\title{
Boron neuron capture therapy using epithermal neutrons for recurrent cancer in the oral cavity and cervical lymph node metastasis
}

\author{
YASUNORI ARIYOSHI ${ }^{1}$, SHIN-ICHI MIYATAKE ${ }^{2}$, YOSHIHIRO KIMURA ${ }^{1}$, TAKESHI SHIMAHARA ${ }^{1}$, \\ SHINJI KAWABATA ${ }^{2}$, KENJI NAGATA ${ }^{3}$, MINORU SUZUKI ${ }^{3}$, AKIRA MARUHASHI $^{4}$, \\ KOJI ONO ${ }^{3}$ and MASASHI SHIMAHARA ${ }^{1}$
}

\begin{abstract}
${ }^{1}$ Division of Surgery 2, Department of Dentistry and Oral Surgery; ${ }^{2}$ Division of Surgery 1, Department of Neurosurgery, Osaka Medical College, 2-7 Daigaku-machi, Takatsuki city, Osaka 569-8686; ${ }^{3}$ Particle Radiation Oncology Research Center, ${ }^{4}$ Medical Physics, Department of Radiation Life Sciences, Research Reactor Institute,
\end{abstract} Kyoto University, Kumatori-cho, Sennan-gun, Osaka 590-0494, Japan

Received March 23, 2007; Accepted June 18, 2007

\begin{abstract}
The purpose of this clinical trial was to evaluate the utility of boron neutron capture therapy (BNCT) using epithermal neutrons for cases of recurrent cancer in the oral cavity, which are not indicated for a conventional treatment modality. We enrolled four patients with local recurrence or metastasis to the regional lymph nodes after completion of initial treatments, including surgery, chemotherapy and radiotherapy. Before receiving BNCT, patients underwent ${ }^{18} \mathrm{~F}$-pbononophenylalanine (BPA) positron emission tomography (PET) examinations to assess the BPA accumulation ratios in tumors and normal tissues. All patients showed at least a tentative partial response, while a marked improvement in quality of life was seen in one patient. Before BNCT, that patient could not be discharged from the hospital because of eating difficulties and malaise; after treatment, he was comfortably discharged. Mild malaise, oral mucositis and alopecia were seen as mild adverse effects; however, no lifethreatening systemic symptoms were observed in any of the cases. Our results suggested that BNCT is a useful treatment modality for recurrent or regionally metastasized oral cancer.
\end{abstract}

\section{Introduction}

Treatment of advanced and/or recurrent oral cancer is a major concern, because the related tumors are known to be

Correspondence to: Dr Yasunori Ariyoshi, Division of Surgery 2, Department of Dentistry and Oral Surgery, Osaka Medical College, 2-7 Daigaku-machi, Takatsuki city, Osaka 569-8686, Japan

E-mail: ora009@poh.osaka-med.ac.jp

Key words: boron neutron capture therapy, epithermal neutron, oral cancer, boronophenylalanine resistant to conventional treatment modalities. We primarily treat patients with advanced squamous cell carcinoma using systemic chemotherapy consisting of Docetaxel and Nedaplatin $(1,2)$. However, because chemotherapy generally induces severe systemic side effects including leukocytopenia, it is difficult to treat patients who have a poor performance status (PS).

Boron neutron capture therapy (BNCT) is a type of tumorcell targeted radiotherapy that has a significantly increased therapeutic ratio in comparison to conventional radiotherapy, without severe systemic side effects. BNCT is based on the nuclear reaction that occurs when boron-10 is irradiated with low-energy thermal neutrons to yield high-linear-energy transfer $\alpha$ particles and recoiling lithium-7 nuclei (3). Clinical interest in BNCT has focused primarily on the treatment of brain tumors and either cutaneous primary or cerebral metastasis from a melanoma; more recently, it has focused on head and neck malignancies $(4,5)$. In the present study, we assessed the usefulness of BNCT for cases of recurrent cancer in the oral cavity and neck metastasis following conventional treatment.

\section{Patients and methods}

Patients. The subjects were 4 patients with recurrent oral cancer and/or cervical neck lymph node metastasis following conventional treatment, including surgery, chemotherapy and radiotherapy (Table I). Each patient signed an informedconsent statement approved by the medical and ethics committees of Kyoto University Research Reactor Institute (KURI) and Osaka Medical College (OMC) prior to enrollment.

Diagnoses of local recurrence and/or metastasis in regional lymph nodes was determined from the results of histopathological and/or cytological examinations, as well as imaging modalities including computed tomography (CT), magnetic resonance images (MRI), and ultrasound (US). After confirmation of recurrence and/or metastasis, the indications for 
Table I. Characteristics of patients.

\begin{tabular}{lccclc}
\hline Case no. & Age/Sex & Location & Histopathological diagnosis & \multicolumn{1}{c}{ Previous treatment } & BNCT fraction \\
\hline 1 & $41 / \mathrm{M}$ & Cervical lymph node & SqCC & Surg, Chem, Rad (39 Gy) & 2 (2 months) \\
2 & $57 / \mathrm{M}$ & Maxilla & SqCC & Surg, Chem, Rad (60 Gy) & 2 (1 month) \\
3 & $67 / \mathrm{F}$ & Maxilla & MC & Surg, Chem, Rad (60 Gy) & 1 \\
4 & $69 / \mathrm{F}$ & Maxilla & AC & Surg, Chem, Rad (60 Gy), Therm & 1 \\
\hline
\end{tabular}

${ }^{a}$ Original tumor site, tongue. M, male; F, female; SqCC, squamous cell carcinoma; MC, mucoepidermoid carcinoma; AC, adenocarcinoma; Surg, surgery; Chem, chemotherapy; Rad, conventional radiotherapy (total doses); Therm, thermotherapy.

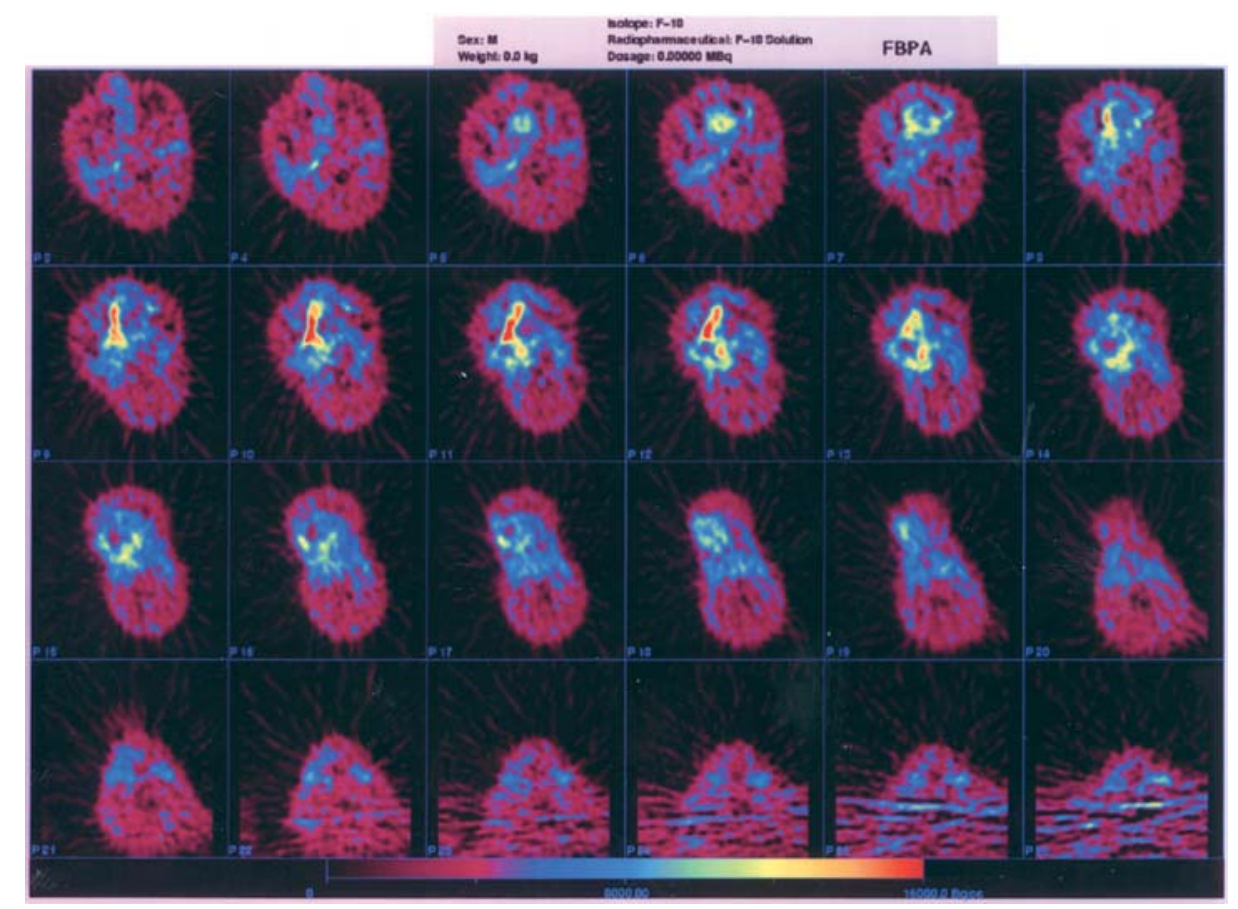

Figure 1. ${ }^{18} \mathrm{~F}$-BPA PET study (case 1). Ratios of ${ }^{18} \mathrm{~F}-\mathrm{BPA}$ accumulation in the area that responsible to tumor and normal tissues were assessed, and the T/N ratio was calculated.

other conventional treatments were assessed. Patients not indicated for another treatment modality were enrolled in the present study and treated with BNCT.

Methods. Prior to BNCT, all patients underwent a fluoride18-labelled p-boronophenylalanine positron emission tomography $\left({ }^{18} \mathrm{~F}-\mathrm{BPA}\right.$ PET) study to assess the distribution of BPA and estimate the boron concentration in the tumor (Fig. 1) $(6,7)$. The tumor/normal $(\mathrm{T} / \mathrm{N})$ tissue ratio of BPA uptake was estimated from those results and dose planning was made according to the $\mathrm{T} / \mathrm{N}$ ratio. Neutron flux was determined using a computer work station equipped with SERA dose planning software (Idaho National Engineering and Environmental Laboratory, Idaho Falls, ID) before the radiation was delivered. The total dose of BPA was $500 \mathrm{mg} / \mathrm{kg}$ body weight. Two hours before neutron irradiation, an intravenous administration of $200 \mathrm{mg} / \mathrm{kg}$ body weight/h of BPA was started. Patients were positioned for neutron radiotherapy in the reactor room and irradiation started after changing the flow rate of BPA to 100 $\mathrm{mg} / \mathrm{kg}$ body weight/h (8). All patients were placed in a sitting position during irradiation. To monitor the boron con- centration in the blood, venous blood samples were obtained every 30 min to $1 \mathrm{~h}$ after BPA was administered until neutron irradiation was completed. ${ }^{10} \mathrm{~B}$ concentrations in the blood were measured by prompt $\gamma$-ray analysis and time vs. ${ }^{10} \mathrm{~B}$ concentration curves were plotted. The boron concentrations from BPA in the tumor and normal tissues were estimated by the $\mathrm{T} / \mathrm{N}$ ratio of ${ }^{18} \mathrm{~F}-\mathrm{BPA}$ on PET. Based on the boron concentration, the neutron fluence rate simulated by the SERA work-station, and factors related to the relative biological effectiveness of the neutron beam and BPA, the total dose delivered to the tumor and normal tissues could be estimated. The duration of irradiation was set to deliver up to 10-15 Gy-Eq to the oral mucosa and as much as possible to the tumor. Here, Gy-Eq (Gy:Gray) means the biologically equivalent $\mathrm{X}$-ray dose that would produce effects equivalent to that of the total BNCT radiation.

Treatment results were assessed by examining clinical features and the results of imaging modalities, including CT and MR imaging. The criteria for responses were defined as follows: complete response (CR), complete disappearance of all clinically evident tumors; partial response (PR), $>50 \%$ 


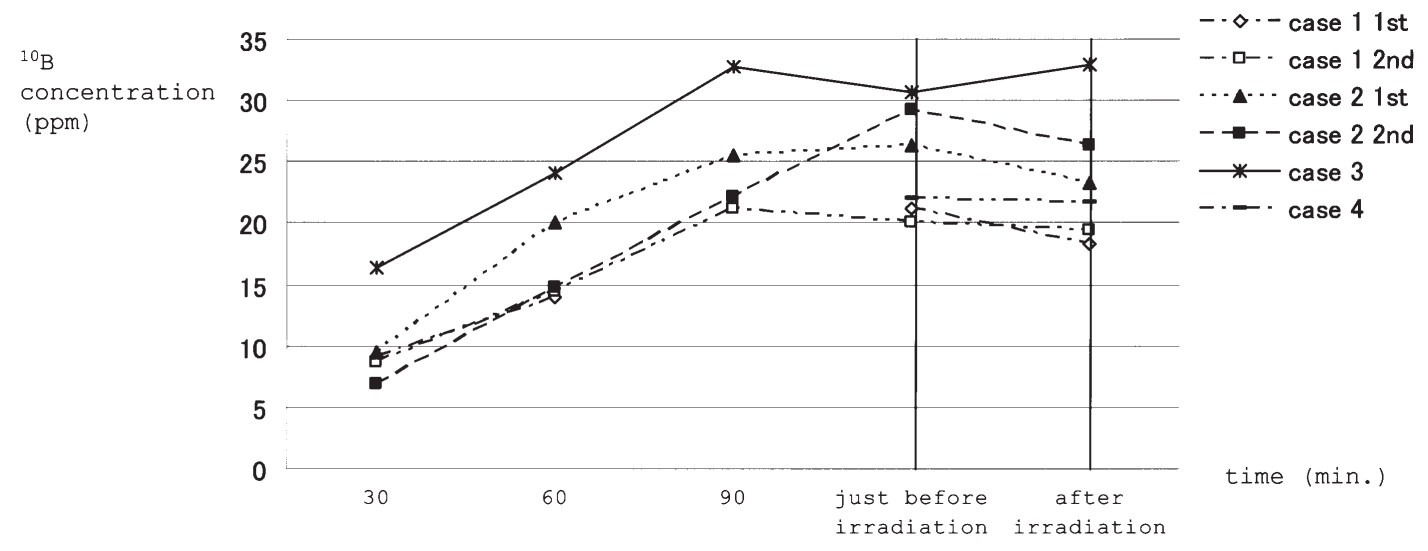

Figure 2. ${ }^{10} \mathrm{~B}$ concentrations in blood. Boron concentrations in blood were monitored using venous blood samples taken before and after irradiation.

Table II. Parameters of BNCT.

\begin{tabular}{lcccccc}
\hline & & \multicolumn{4}{c}{ Dose (Gy-Eg) } \\
\cline { 4 - 6 } Case & $\begin{array}{c}\text { T/N ratio of } \\
{ }^{18} \text { F-BPA }\end{array}$ & $\begin{array}{c}\text { Irradiation } \\
\text { time (min) }\end{array}$ & $\begin{array}{c}\text { Skin } \\
\text { surface }\end{array}$ & $\begin{array}{c}\text { Oral } \\
\text { mucosa }\end{array}$ & $\begin{array}{c}\text { Tumor peak } \\
\text { (depth from skin surface, cm) }\end{array}$ & $\begin{array}{c}\text { Minimum tumor dose } \\
\text { (depth from skin surface, cm) }\end{array}$ \\
\hline 1 & 4.0 & 90 & 4.9 & 14.4 & $39.1(1.8)$ & $15.0(7.0)$ \\
& - & 75 & 2.9 & 9.5 & $25.5(2.5)$ & $10.3(7.0)$ \\
2 & 3.4 & 41 & 3.2 & 9.1 & $21.6(2.0)$ & $9.1(6.0)$ \\
& - & 60 & 4.6 & 14.4 & $20.1(2.5)$ & $8.9(6.0)$ \\
3 & 2.2 & 36 & 3.7 & 15.3 & $24.8(2.5)$ & $22.0(4.0)$ \\
\hline
\end{tabular}

reduction in the sum of the two largest perpendicular diameters of each measurable disease site, with no appearance of new lesions or progression of any existing lesions; progressive disease (PD), at least a $25 \%$ increase in tumor area or the appearance of new lesions; no change (NC), all other outcomes. Adverse effects were assessed using the National Cancer Institute Common Toxicity Criteria (NCI-CTC ver.2.0).

\section{Results}

BNCT parameters. The $\mathrm{T} / \mathrm{N}$ ratios of ${ }^{18} \mathrm{~F}-\mathrm{BPA}$, which were assessed in the PET examinations, ranged from 2.2 to 4.0 . The fraction of irradiation was 2 in two cases (cases 1 and 2) and 1 in two cases (cases 3 and 4). The duration of neutron irradiation was from 26 to $90 \mathrm{~min}$. The concentration of ${ }^{10} \mathrm{~B}$ just before and just after irradiation ranged from 30.7 to $20.0 \mathrm{ppm}$ and 32.9 to $18.3 \mathrm{ppm}$, respectively (Fig. 2). The minimum tumor doses (usually at the deepest part of the tumor) ranged from 22.0 to $8.9 \mathrm{~Gy}$-Eq. The maximum doses for the oral mucosa and skin surface ranged from 15.3 to 9.1 Gy-Eq and 7.2 to 2.9 Gy-Eq, respectively (Table II).

Clinical response, adverse effects, PS and outcome. Clinical response was determined to be PR in 3 cases and PD in 1
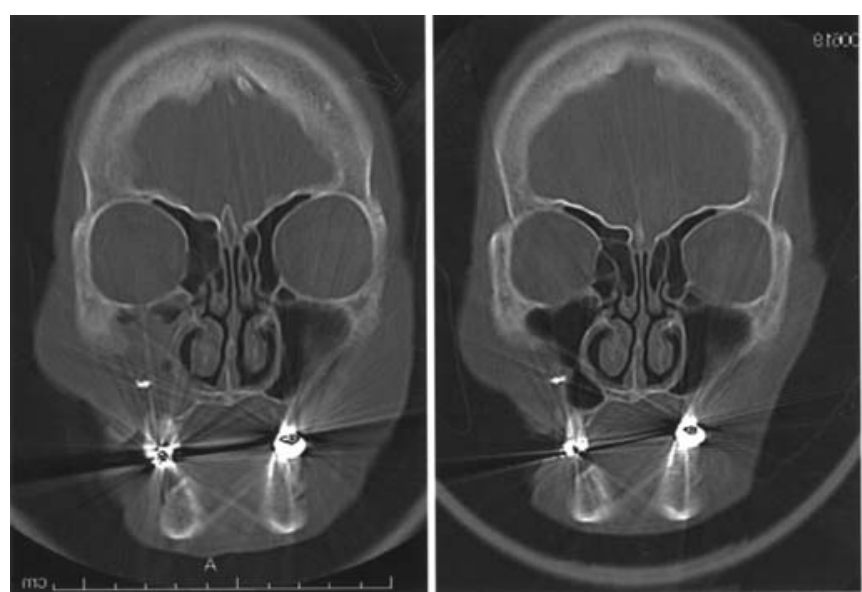

Figure 3. Case presentation (case 3). Left, prior to irradiation; right, 5 months after irradiation. A CT examination revealed a marked decrease in tumor size. Before irradiation (left), the right antrum was filled with a dense and soft tissue mass. Following BNCT (right), the tumor mass was decreased in size and the right antrum appeared as a pneumatic space.

(Figs. 3 and 4). As for systemic adverse effects, mild fatigue was detected in all cases, though apparent hematological toxicities were not seen. Locally, mucositis appearing within 

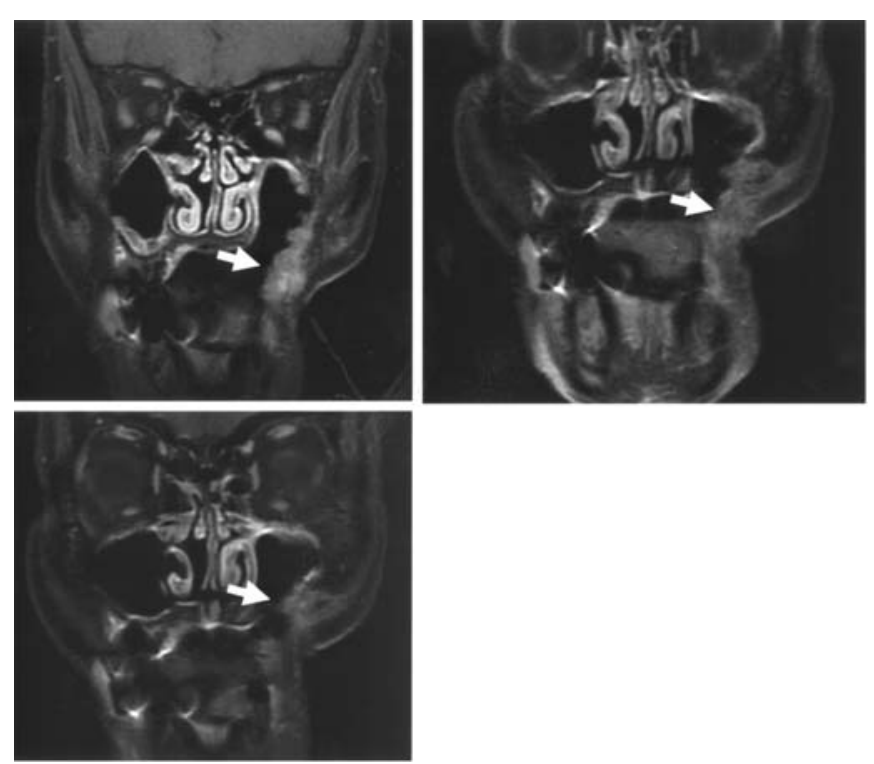

Figure 4. Case presentation (case 2). Top left, prior to irradiation; top right, just prior to second irradiation; bottom, 3 months after completion of BNCT. An enhanced mass lesion was seen in the left buccal area in all three phases (arrows). Although no apparent decrease in tumor size was demonstrated just before the second irradiation, marked tumor downsizing was noted 3 months after the completion of BNCT.

1 week after irradiation and continuing for 3-5 weeks was the most severe adverse effect. No skin defects that had a direct relation to BNCT were seen in the irradiated fields, except in the case 1 patient, whose skin already showed tumor invasion before BNCT. PS was improved in 3 of 4 cases, while the remaining patient, who did not complain of any symptoms including severe pain before irradiation, showed no change (Table III). The most apparent improvement in PS was seen in the case 1 patient, who had been hospitalized because of severe pain and difficulties in eating before BNCT. His neck mass was markedly downsized and pain was relieved, and he was tentatively discharged from the hospital after BNCT. Two of 4 patients (cases 3 and 4 ) were alive with evidence of disease during follow-up periods ranging from 6 to 12 months. One patient (case 1) died from tumor re-growth followed by aspiration pneumonia 2 months after completion of BNCT, and the remaining patient (case 2) died from local tumor regrowth 12 months after completion of BNCT.

\section{Discussion}

Results of the first clinical trial of BNCT for head and neck cancer including oral cancer were reported in 2004 (5). In 2005, we began BNCT at our institution, mainly for patients with advanced non-resectable and recurrent oral cancer. In general, such patients suffer from severe pain, difficulty with eating that induces malnutrition, difficulty with speaking, and poor PS. For recurrent cancer patients, it is controversial which kind of treatments should be chosen, radical or palliative. To resolve this issue, both the clinical response, including prognosis, and improvement of PS should be discussed when a treatment modality for these patients is decided.

BNCT is a novel treatment modality for these patients, because it offers the possibility of inducing radical rather than palliative treatment outcomes. Prior to administering BNCT to oral cancer patients, it is important to discuss the protocol, as conventional BNCT for a brain tumor has a number of problems, such as lack of neutron penetration, especially in patients with deep-seated tumors, an insufficient contrast in boron concentration between tumors and normal tissues, an absolute lack of boron in tumor tissues, and an uncertain estimation of neutron flux captured by the ${ }^{10} \mathrm{~B}$ atoms in tumor cells (9). In the present study, we assessed macroscopic treatment effects and investigated clinical problems associated with BNCT administered for recurrent oral cancer and neck metastasis following conventional treatment.

BNCT indications were assessed using ${ }^{18} \mathrm{~F}-\mathrm{BPA}$ PET, which provides an accurate assessment of BPA accumulation

Table III. Clinical results.

\begin{tabular}{|c|c|c|c|c|}
\hline Case & Clinical response & Adverse effects & Improved PS & $\begin{array}{l}\text { Outcome (duration of BNCT } \\
\text { and time of outcome determination) }\end{array}$ \\
\hline 1 & PD & $\begin{array}{l}\mathrm{Fa}(1) \\
\mathrm{Mu}(2)\end{array}$ & $2+$ & Died (4 months) \\
\hline 2 & PR & $\begin{array}{l}\mathrm{Fa}(1) \\
\mathrm{Mu}(1)\end{array}$ & +- & Died (13 months) \\
\hline 3 & PR & $\begin{array}{ll}\mathrm{Fa} & (1) \\
\mathrm{Al} & (1)\end{array}$ & $1+$ & AD (13 months) \\
\hline 4 & PR & $\begin{array}{l}\mathrm{Fa}(1) \\
\mathrm{Mu}(2)\end{array}$ & $1+$ & $\mathrm{AD} \quad$ (7 months) \\
\hline
\end{tabular}

Clinical response: PD, progressive disease; PR, partial response. Adverse effects: Fa, Fatigue; Mu, mucositis; Al, alopecia. Improved PS: $2+$, improved by 2 grades or more; $1+$, improvement of 1 grade; +-, no change; -, exacerbation. Outcome: AD, alive with disease. 
and distribution before irradiation. Kato et al (5) reported that a head and neck tumor with a $\mathrm{T} / \mathrm{N}$ ratio of $<2.5$ or a tumor in a location deep under the skin surface should not be recommended for BNCT. In the present 4 cases, the $\mathrm{T} / \mathrm{N}$ ratios were 4.0, 3.4, 2.2 and 2.4 , respectively. The latter 2 (cases 3 and 4) showed low $\mathrm{T} / \mathrm{N}$ values according to the criteria of Kato et al; however, relatively good responses were obtained. The tumors in those patients were superficially located near the skin surface and their positions allowed for relatively easy access during irradiation. Conversely, cases that showed higher $\mathrm{T} / \mathrm{N}$ values (cases 1 and 2 ) had unfortunate outcomes within a 12-month follow-up period. The clinical effects seen in the present study suggest that tumor location and patient performance are important, in addition to the $\mathrm{T} / \mathrm{N}$ ratio obtained by ${ }^{18} \mathrm{~F}$-BPA PET. Patient positioning during irradiation has a strong influence on the effects of BNCT. Notably, patients with a neck dissection, such as case 1 , find it difficult to stretch their neck, while the shoulder interferes with accurate positioning. To improve the effects of BNCT, it is necessary to modify patient positioning for each case.

Kato et al (5) indicated that BNCT represents a new and promising treatment approach, even for large or far advanced head and neck malignancies, because it can induce a remarkable reduction of huge tumors, improve QOL, and has very mild side effects. In the present study, 3 of 4 patients were categorized as PR and the remaining patient as PD. We considered that the overall clinical effects were excellent for our patients, who had recurrent disease and had already undergone other conventional treatment modalities including surgery, chemotherapy and radiotherapy. In the PD case (case 1), a tentative tumor decrease was seen; however, tumor re-growth occurred and the patient died 4 months after the first course of BNCT. That tentative decrease in tumor mass led to an improvement in PS, namely, pain relief, and as a result the patient was discharged temporarily from the hospital. In both of the fatal-outcome cases (cases 1 and 2), tumor re-growth occurred in the part deep from the skin surface. These results suggest that the minimum tumor dose was not enough to destroy the tumor cells at the areas of recurrence. Between the tumor re-growth cases (cases 1 and 2) and no-re-growth cases, there was no essential difference in estimated minimum tumor dose. Thereafter, the recurrence might be ascribed to heterogeneous distribution of boron compounds in the re-growth cases (strategies for overcoming this problem are discussed later). Recurrence in these cases occurred at the deepest part of the tumor, where the absorbed dose in tumor tissue was the lowest.

Kato et al (5) stated that one of the merits of BNCT is its indication for recurrent or metastatic cases that have already received a full dose of radiotherapy. All of the present patients had experienced conventional radiotherapy before undergoing BNCT. Three of the 4 had already received 60 Gy of radiation in the same field that received BNCT, and the remaining case (case 1) had received $39 \mathrm{~Gy}$. As a result, following BNCT, there was a concern regarding perforation of the covering skin, osteoradionecrosis or rupture of vital vessels like the carotid artery. At present, no such severe complication has been seen in the patients. In one patient who died (case 1), the tumor had already invaded the skin, and skin perforation was evident before performing BNCT.
Skin perforation was also seen in case 2 . In this case, although the tumor showed good response to BNCT, aggressive tumor re-growth was seen eight months after irradiation, and tumor invasion to the skin followed by perforation occurred. The irradiated doses to the skin were not significantly different between cases 1 and 2 (fatal outcomes) and 3 and 4 (good outcomes). These results suggest that previous irradiation should not prohibit the administration of BNCT. On the other hand, because the follow-up period is limited, we can not predict whether osteoradionecrosis of the maxilla and adjacent bones will occur in the case 3 and 4 . As a result, we can make no definite conclusion as to whether previous irradiation should prohibit the administration of BNCT or restrict its dose. To resolve these issues, long-term follow-up of a large number of cases is required.

From a clinical point of view, it is important to assess adverse effects as well as clinical response. Regarding brain tumors, one of the authors of this paper (Miyatake et al) (9) reported that all of their patients exhibited alopecia, and no acute brain swelling or consciousness disturbance occurred. As for head and neck tumors, Kato et al (5) noted that there were few side effects such as transient mucositis and alopecia, and all side effects were less than grade- 2 by NCI-CTC. In the present patients, no severe systemic adverse effects including malaise, anemia or leukocytopenia were seen. On the other hand, mucositis, which can cause difficulties with eating and degrade QOL, occurred and continued for 3 to 4 weeks in all patients. In addition, in the patient with maxillary cancer and the case of a metastatic neck lymph node, localized alopecia was seen. Those systemic and localized adverse effects suggest that, except for the possibility of mucositis, BNCT has more benefits for patients than conventional treatments, including chemotherapy and radiotherapy.

Selection of the boron delivery agents is also an important factor for BNCT (4). Previous clinical trials $(5,9)$ used a combination of BPA and sodium borocaptate (BSH). Ono et al (8) reported that accumulation of BPA occurred in cycling tumor cells but not in quiescent cells of solid tumors, and combination with BSH was one of the solutions for the problem of heterogeneous micro-distribution of BPA $(10,11)$. Further, Ono et al (8) considered that the new BPA injection and neutron irradiation protocol was able to overcome the difficulties of BPA. In the present study, we used the same new BPA injection and neutron irradiation protocol, and good results were obtained. However, the number of cases was quite limited; thus, further investigation is required to determine the utility of this new BPA protocol for oral cancer. Other boron compound infusion methods for treatment of brain tumors including long-term infusion of BPA (12) and intracarotid injection of compounds (13) have been reported. Nevertheless, the indications for those methods in regard to oral cancer including metastatic neck nodes should also be investigated.

\section{Acknowledgements}

This study was supported in part by a Grant-in-Aid for Scientific Research (C) (No.18592224) to M.S. from Japan Society for the Promotion of Science and by a grant from the Takeda Science Foundation to Osaka Medical College. 


\section{References}

1. Ariyoshi Y, Shimahara M, Shimahara T, Tanigawa N and Tsuji M: Chemotherapy for advanced squamous cell carcinoma of the oral cavity with Nedaplatin and Docetaxel. In: Proceedings of the 5th Congress of the International College for Maxillofacial Surgery. Mogi K and Negishi A (eds). pp189-193, 2005.

2. Ariyoshi Y, Shimahara M, Kurisu Y and Tsuji M: Docetaxel and Nedaplatin chemotherapy for advanced oral squamous cell carcinoma: a case report. Int J Oral Med Sci 3: 49-53, 2004.

3. Coderre JA and Morris GM: The radiation biology of boron neutron capture therapy (Review). Radiation Res 151: 1-18, 1999.

4. Barth RF, Conderre JA, Vincente MGH and Blue TE: Boron neutron capture therapy of cancer: current status and future prospects. Clin Cancer Res 11: 3987-4002, 2005.

5. Kato I, Ono K, Sakurai Y, et al: Effectiveness of BNCT for recurrent head and neck malignancies. Appl Radiat Isotopes 61: 1069-1073, 2004

6. Imahori Y, Ueda S, Ohmori Y, et al: Positron emission tomography-based boron neutron capture therapy using boronophenylalanine for high-grade gliomas: Part I. Clin Cancer Res 4: 1825-1832, 1998.

7. Imahori Y, Ueda S, Ohmori Y, et al: Positron emission tomography-based boron neutron capture therapy using boronophenylalanine for high-grade gliomas: Part II. Clin Cancer Res 4: 1833-1841, 1998.
8. Ono K, Masunaga S, Kinashi Y, et al: Neutron irradiation under continuous BPA injection for solving the problem of heterogenous distribution of BPA. In: Advances in Neutron Capture Therapy. Takamatsu pp27-30, 2006.

9. Miyatake S, Kawabata K, Kajimoto Y, et al: Modified boron neutron capture therapy for malignant gliomas performed using epithermal neuron and two boron compounds with different accumulation mechanisms: an efficacy study based on finding on neuroimages. J Neurosurg 103: 1000-1009, 2005.

10. Ono K, Masunaga S, Kirihashi Y, et al: Radiobiological evidence suggesting heterogeneous microdistribution of boron compounds in tumors: its relation to quiescent cell population and tumor cure in neutron capture therapy. Int $\mathrm{J}$ Radiat Oncol Biol Phys 34: 1081-1086, 1996.

11. Ono K, Masunaga S, Suzuki M, Kinashi Y, Takagi M and Akabishi M: The combined effect of boronoophenylalanine and borocaptate in boron neutron capture therapy for SCC tumors in mice. Int J Radiat Oncol Biol Phys 43: 431-436, 1999.

12. Morris GM, Micca PL, Nawrocky MM, Weissfloch LE and Coderre JA: Long-term infusion of p-boronophenylalanine for boron neutron capture therapy: evaluation using rat brain tumor and spinal cord models. Radiat Res 158: 743-752, 2002.

13. Barth RF, Yang W, Rotaru JH, et al: Boron neutron capture therapy of brain tumors; enhanced survival and cure following blood-brain barrier distribution and intracarotid injection of sodium borocaptate and boronophenylalanine. Int J Radiat Oncol Biol Phys 47: 209-218, 2000. 